most powerful, is extended to testing composite hypotheses where the forms of the distribution functions are entirely unknown (continuity is assumed) and where tests must be based on the order relations among the observations. Thus a general method for treating problems of this character is obtained. For the problem of two samples (Wald and Wolfowitz, Annals of Mathematical Statistics, June, 1940) the resultant statistic is $\prod_{i} l_{j} l_{j} /\left(l_{j}\right)$ !, where $l_{j}$ is the length of the $j$ th run. Its logarithm is asymptotically normally distributed. The result is immediately extensible to the problem of $k$ samples. For the problem of independence (Hotelling and Pabst, Annals of Mathematical Statistics, March, 1936) a similar statistic is obtainable which differs from the commonly used rank correlation coefficient. The method used to prove the logarithms of these statistics asymptotically normally distributed is applicable to proving the asymptotic normality of a large class of functions of partitions of an integer, of functions of sequences where the subsequences of odd and even numbered elements are themselves partitions of different integers of fixed ratio, and to similar problems (Received January 13, 1941.)

\title{
Theory of Numbers
}

173. Paul Erdös and Joseph Lehner: On the distribution of the number of summands in the partitions of a positive integer.

Let $p_{k}(n)$ denote the number of partitions of $n$ into not more than $k$ summands. Then for $k=n^{1 / 2}(\log n / C)+x n^{1 / 2}, C=\pi(2 / 3)^{1 / 2}, p_{k}(n) / p(n) \sim \exp \{-2 \exp (-C x / 2) / C\}$, where $p(n)$ is the number of unrestricted partitions of $n$. For $k=o\left(n^{1 / 3}\right), p_{k}(n)$ $\sim C_{n-1, k-1} / k$ !, uniformly in $k$. Let $P(n)$ be the number of partitions of $n$ into different summands. Then for "almost all" partitions, the number of summands in a given partition not exceeding $x n^{1 / 2}$ lies between $2 n^{1 / 2}\{\log 2 /(1+\exp (-D x))\} / D \pm \epsilon n^{1 / 2}$, $D=\pi(1 / 3)^{1 / 2}$. The methods used are elementary in character. (Received January 23, 1941.)

174. Gordon Pall: The construction of positive ternary quadratic forms.

A method is developed of writing down quickly the reduced integral positive ternary quadratic forms of a given determinant, order, or genus. (Received January $30,1941$.

175. H. A. Rademacher: Ramanujan's identities under modular transformations.

If the Ramanujan identities which exhibit the divisibility of $p(5 n+4)$ and $p(7 n+5)$ by 5 and 7 , respectively, are expressed in terms of the Dedekind function $\eta(\tau)$, they can be subjected to modular transformations. Each of the two identities goes over into a new one, which is noteworthy because of the occurrence of the Legendre residue symbol. These new identities lead to a direct proof of the Ramanujan identities. The same procedure can be applied to an identity given by Zuckerman, involving $p(13 n+6)$. Other identities, proved by Watson and Zuckerman, lead to modular equations of "level" (Stufe) 5 and 7. (Received January 24, 1941.)

\section{Topology}

176. F. B. Jones: Monotonic collections of peripherally-separable connected domains.

It is shown that: In a locally connected metric space, every monotonic collection of 
peripherally-separable connected domains contains a countable subcollection running through it. Weakening the hypothesis of the theorem in any one respect results in a false proposition. A few simple applications are indicated. (Received January 24, 1941.)

\section{Shizuo Kakutani: A generalization of Brouwer's fixed-point} theorem.

A generalization of Brouwer's fixed-point theorem (concerning a continuous pointto-point mapping $\phi(x)$ of a finite dimensional simplex $S$ into itself) is obtained for the case of a point-to-set mapping $\Phi(x)$, when the image $\Phi(x)$ of each point is a closed, convex set contained in or equal to $S$. Instead of the continuity of $\phi(x)$, the upper semicontinuity of $\Phi(x)$ is assumed. The fixed-point theorems of J. von Neumann in his theory of games (Mathematische Annalen, vol. 100 (1928)) and mathematical economics (Ergebnisse eines Mathematischen Kolloquiums, vol. 8 (1937)) are easy consequences of this result. In order to prove the theorem, the fixed-point theorem of Brouwer must be assumed. (Received January 22, 1941.)

\section{J. L. Kelley: A theorem on transformations.}

Let $f(X)=Y$ ke a monotone interior transformation of a compact metric space and suppose $\operatorname{dim} Y<+\infty$. Further suppose $\operatorname{diam} f^{-1}(y) \geqq d>0$ for all $y \in Y$. Then there exists a closed, totally disconnected subset $X_{0}$ of $X$ such that $f\left(X_{0}\right)=Y$. As a result it is shown that if there exists a Knaster continuum (that is, an indecomposable continuum every subcontinuum of which is decomposable) of dimension greater than 1 , then there exists a Knaster continuum of infinite dimension. (Received December 30, 1940.)

\section{R. C. Stephens: On abstract mappings.}

Following the notation of Chittenden, a space $(P, \phi)$ denotes an abstract set $P$ and a relation between subsets of $P$. A mapping $T$ of $(P, \phi)$ onto $(Q, \phi)$ will be called a $\phi$-mapping provided that for every subset $E$ of $Q$, the inverse transformation $T^{-1}$ satisfies the relation $\phi T^{-1}(E)=T^{-1} \phi(E)$. In this paper, theorems are established for $\phi$-mappings with $\phi$ having various simple properties. These results are then applied to standard operators. The relationship of $\phi$-mappings to interior transformations is shown. (Received January 20,1941.)

\section{Hassler Whitney: Geometric methods in combinatorial topology. Preliminary report.}

Singular cycles in a polyhedron $P$ give a geometric content to the theory of cycles in a corresponding complex $K$. "Hypercycles" in $P$ are defined corresponding to cocycles in $K$. A hyperchain $\rho$ is, essentially, a homomorphism, into a coefficient group $G$, of singular cycles $C$ whose boundaries do not touch the "nucleus" $N_{\rho}$ of $\rho$, and which themselves do not touch the "nuclear boundary" $N_{\rho}^{\prime}\left(N_{\rho}^{\prime} \subset N_{\rho}\right)$. The "hyperboundary" $\rho^{\prime}$ of $\rho$ is easily defined: $N_{\rho^{\prime}}=N_{\rho}^{\prime}, N_{\rho^{\prime}}^{\prime}=0$. Any $\rho^{r} \cdot C^{r}$ is an element of $G$. If $K^{\prime}$ is the first derived of $K$, to each oriented $\sigma$ corresponds a $\rho_{\sigma}$, whose nucleus is the set of closed cells of $K^{\prime}$ touching $\sigma$ only at its center, and with "orientation" that of $\sigma$. Then $\rho_{\sigma} \cdot \sigma=1$. In an orientable manifold $M^{n}=K$, a cycle $C^{r}$ of $K$ corresponds to a $\rho^{n-r}$ with nucleus $C^{r}$, and a chosen orientation of cells $\sigma^{n-r}$ normal to $C^{r}$. If $\rho^{r}$ and $\rho^{8}$ are in "general position," their "product" gives a $\rho^{r+s}=\rho^{r} \smile_{\rho^{s}} ; N_{\rho^{r+s}}=N_{\rho^{r}} \cap N_{\rho^{s}}$. Also $\rho^{s}-C^{r+s}=C^{r}$. In $M^{n}$, passing to cycles, both products give ordinary intersections. (Received January 25, 1941.) 
181. G. T. Whyburn: On mappings into the circle.

If $f(x)$ is a continuous mapping of a metric space $X$ into the unit circle $S$ of the complex plane, the property of a subset $Y$ of $X$ to have $f$ not equivalent to 1 (in the Eilenberg sense) on $Y$ is inducible. Thus if $X$ is compact and $f$ is not equivalent to 1 on $X$, there exists a compact subset $Y$ of $X$ on which $f$ is irreducibly not equivalent to 1 . Further, any such set $Y$ is a continuum not separated by any proper subcontinuum. Hence the property of having $f \sim 1$ is $E_{0}$-extensible for compact sets $X$; that is, if $f \sim 1$ on each $E_{0}$-set of every component of $X$, then $f \sim 1$ on $X$. In particular, property (b): to have every continuous mapping into the circle equivalent to 1 , is $E_{0}$-extensible and reducible. Using this result it is shown directly that the number $b(Y)$ is cyclicly additive for compact sets $X$, that is, $b(X)=\sum b\left(E_{\alpha}\right)$ where the $E_{\alpha}$ are the simple links of the components of $X$ and where, in general, $b(Y)$ denotes the rank of the factor group $S^{Y} / P(Y)$ where $S^{Y}$ is the group of all mappings into $S$ and $P(Y)$ is the subgroup of all elements of $S^{Y}$ which are equivalent to 1 on $Y$. (Received January 21,1941.) 